\title{
Genetic Diversity and Population Structure Analysis of Dalbergia Odorifera Germplasm and Development of a Core Collection Using Microsatellite Markers
}

\author{
Fu-Mei Liu ${ }^{1,2}{ }^{1}$, Ning-Nan Zhang ${ }^{1}$, Xiao-Jin Liu ${ }^{1}$, Zeng-Jiang Yang ${ }^{1}$, Hong-Yan Jia ${ }^{2,3}$ and \\ Da-Ping $\mathrm{Xu}^{1, *}$ \\ 1 Research Institute of Tropical Forestry, Chinese Academy of Forestry, Longdong, Guangzhou 510520, China; \\ liufumei1115@163.com (F.-M.L.); ningnanzhang@126.com (N.-N.Z.); xjliucaf@163.com (X.-J.L.); \\ yzengjiang@126.com (Z.-J.Y.) \\ 2 The Experimental Centre of Tropical Forestry, Chinese Academy of Forestry, Pingxiang 532600, China; \\ rlzxjhy@163.com \\ 3 Guangxi Youyiguan Forest Ecosystem Research Station, Pingxiang 532600, China \\ * Correspondence: gzfsrd@163.com; Tel.: +86-20-8703-1037
}

Received: 26 February 2019; Accepted: 1 April 2019; Published: 6 April 2019

\begin{abstract}
Dalbergia odorifera T. Chen (Fabaceae) is a woody tree species indigenous to Hainan Island in China. Due to its high medicinal and commercial value, this tree species has been planted over $3500 \mathrm{ha}^{2}$ in southern China. There is an urgent need for improvement of the D. odorifera germplasm, however, limited information on germplasm collection, conservation, and assessment of genetic resources is available. Therefore, we have built a database of 251 individuals collected across the whole of southern China, which included 42 wild trees and 210 cultivated trees, with the following objectives. (1) Evaluate genetic diversity and population structure of the database using 19 microsatellite markers and (2) develop a core collection for improvement and breeding programs. Totally, the 19 microsatellite markers harbored 77 alleles across the database with the polymorphic information content (PIC) ranging from 0.03 to 0.66 . Medium genetic diversity level was inferred by Nei's gene diversity (0.38), Shannon's information index (0.65), and observed (0.33) and expected heterozygosity (0.38). Structure analysis showed that four was the optimum cluster size using the model-based Bayesian procedure, and the 251 D. odorifera individuals were grouped into five populations including four pure ones (RP1-4) and one mixed one (MIX) based on their maximum membership coefficients. Among these populations, the expected heterozygosity varied from 0.30 (RP3) to 0.38 (RP4). Analysis of molecular variance (AMOVA) showed $11 \%$ genetic variation existed among populations, and moderate population differentiation was inferred by the matrix of pairwise Fst (genetic differentiation among populations), which was in the range of 0.031 to 0.095 . Moreover, a core collection of $31 \mathrm{D}$. odorifera individuals including six wild and 25 cultivated trees was developed, which was only $12.4 \%$ of the database but conserved the whole genetic diversity. The results of this study provided additional insight into the genetic structure of the large $D$. odorifera germplasm, and the core collection will be useful for the efficient and sustainable utilization of genetic resources, as well as efficient improvement in breeding programs.
\end{abstract}

Keywords: Dalbergia odorifera T. Chen; genetic diversity; core collection; SSR marker; Rosewood; conservation

\section{Introduction}

Dalbergia odorifera T. Chen, formerly named Dalbergia hainanensis Merr. et Chun, is a semideciduous perennial woody tree species of high medicinal and commercial value. The heartwood of its root and 
stem is a traditional Chinese medicine, named "Jiangxiang", which plays crucial roles in cardiovascular treatments [1], diabetes [2], blood disorders, etc. [3,4] as it contains sets of complex and useful chemical components [5-9]. The heartwood of this species, also named "Hualimu" or "Huanghuli" (Chinese name), is a precious fragrant rosewood with extremely high value in the luxury furniture and craft markets in China. Often the most highly valued timber trees are the most threatened species in their native habitats [10]. For example, D. odorifera-an indigenous to Hainan Island in southern China and is confined to a relatively narrow tropical geographic area [11] - that has been overexploited for a long time and has been listed on the IUCN (International Union for Conservation of Nature's) red list by World Conservation Monitoring Centre (WCMC) since 1998 [12]. The resource has also been promoted to a second-grade state protected species by the Chinese government. It is now in danger of extinction resulting from illegal logging. Currently, only a limited number of plants exist in the remaining forest fragments [11,13].

Due to its high medicinal and commercial value, D. odorifera was introduced to subtropical areas of Guangdong, Guangxi, and Fujian provinces in China in the 1950s. After several decades, the introduced trees exhibited a satisfactory growth performance, and, at most sites, has even formed valuable heartwoods, which take approximately 50 years to mature in the wild [14]. Recently, more and more farmers planted $D$. odorifera trees because of its ecological and economic importance. Moreover, the plantation area of this species has exceeded $3500 \mathrm{ha}^{2}$ in southern China. There is an urgent need for improvements of the $D$. odorifera germplasm, particularly for higher yield of heartwood in a shorter harvest cycle. Such improvements are based on the germplasm collection, conservation, and assessment of genetic resources.

Germplasm is the basis of plant breeding programs [15]. During the last several decades, there has been remarkable progress in D. odorifera germplasm collection [16-18]. Eventually, a dataset of 251 individuals has been collected and conserved in the Research Institute of Tropical Forestry (Chinese Academy of Forest, CAF) and in Guangxi Youyiguan Forest Ecosystem Research Station at the Experimental Center of Tropical Forestry (CAF). These individuals came from all over southern China, covering its native habitat (Hainan Island) and introduced sites (Guangdong, Guangxi, Hunan, and Fujian provinces). However, the original information on these introduced germplasm is confusing due to frequently disordered introductions within and among different non-native regions. Little is known about the genetic diversity and structure of the whole collection. These issues have limited the efficient and sustainable utilization of the genetic resources. Hence, a core collection has been proposed to deal with this issue.

A core collection is a subset of the whole germplasm collection, and aims to create a uniform representation of the original genetic space with equal weights across this space [19]. A good core collection is one that has no redundant accessions, is small enough to be easily managed, and represents the total genetic diversity [20]. Notably, core collections have been developed for many tree species, including apple (Malus domestica Borkh) [21,22], avocado (Persea americana Mill.) [23], and chestnut (Castanea sativa Mill.) [24]. However, no core collection has been developed for D. odorifera yet. Additionally, information on the genetic diversity and genetic variation of plant species has been well demonstrated using molecular markers [25-28], which are essential to core collection, germplasm identification, and breeding purposes [29]. Therefore, we conducted a comprehensive survey on genetic diversity and genetic variation for the database of $251 \mathrm{D}$. odorifera individuals using a set of 19 microsatellite markers, and developed a core collection using PowerCore software [30]. The main objectives of this study were to (1) evaluate genetic diversity and population structure of the D. odorifera germplasm and (2) develop a core collection conserving the entire diversity for improvement and breeding programs. The findings of this study will provide a useful resource as well as guidance for better germplasm utilization in genetic improvement, and serve as a database for identification, parentage, and traceability purposes. 


\section{Materials and Methods}

\subsection{Plant Materials and DNA Extraction}

A total of 251 individuals, including 42 wild trees and 209 cultivated trees, was collected from the native habitat and introduced sites of D. odorifera across the southern China (Table S1). Among the cultivated individuals, 62 came from Hainan Island, 69 from Guangxi province, 52 from Guangdong province, 20 from Fujian province, and 6 from Hunan province. Permission for leaf sample collection was obtained from the local managers. Ten young leaves were collected from each individual and sealed in plastic bags with desiccants. Total genomic DNA was extracted for each sample using the Hi-DNAsecure Plant Kit (Tiangen, Beijing, China) according to the manufacturer's instructions. The quality and quantity of DNAs were determined by NanoDrop 2000 (Thermo Scientific, Wilmington, DE, USA).

\subsection{PCR and Capillary Electrophoresis}

A set of 19 microsatellite markers was developed in our previous study [13] and used in the present study (Table S2). Subsequently, PCR reactions with the designed primers [13] were carried out using DNAs for all the 251 samples. PCR reactions were of $15 \mu \mathrm{L}$ final volume, containing $10.25 \mu \mathrm{L}$ water, $1.5 \mu \mathrm{L}$ 10× DNA polymerase buffer, $1.5 \mu \mathrm{L} \mathrm{MgCl}_{2}(25 \mathrm{mM}), 0.3 \mu \mathrm{ldNTPs}(10 \mathrm{mM}$ each), $0.15 \mu \mathrm{L}$ of each primer at $10 \mu \mathrm{M}, 0.3 \mu \mathrm{L}$ Taq polymerase at 5 units/ $\mu \mathrm{L}$ (TaqUBA), and $1 \mu \mathrm{L}$ of genomic DNA (40-50 ng). Then, 35 cycles of $94{ }^{\circ} \mathrm{C}$ for $15 \mathrm{~s}$, appropriate annealing temperature for $15 \mathrm{~s}$, and $72{ }^{\circ} \mathrm{C}$ for $30 \mathrm{~s}$ were performed, following the predenaturation at $94{ }^{\circ} \mathrm{C}$ for $3 \mathrm{~min}$. All the PCR reactions were repeated at least once. Their diluted PCR products were mixed with $12.5 \mathrm{Hi}$-Di formamide and $0.25 \mu \mathrm{L}$ of size standard (Shanghai Generay Biotech Co., Ltd, Shanhai, China), and were then separated by capillary electrophoresis and genotyped with an ABI 3730 Genetic Analyzer (Applied Biosystem, Foster, CA, USA) at Shanghai Generay Biotech Co., Ltd., Shanghai, China. Peak identification and fragment sizing were done using Gene Mapper v4.0 (Applied Biosystems) with the default settings.

\subsection{Population Structure}

The genetic structure of the investigated database was analyzed using STRUCTURE 2.0 [31]. The number of discontinuous $\mathrm{K}$ was estimated from one to ten with twenty iterations. Both the length of burn-in period and value of MCMC (Markov chain Monte Carlo) were set to 100,000 times [32]. The admixture model was used with correlated allele frequencies, the options "popinfo" and "popflag" were both set to zero to consider that the sampled individuals were of unidentified origin. Next, the optimum value of cluster $(\mathrm{K})$ was harvested online according to the highest $\ln \mathrm{P}(\mathrm{D})$-derived $\Delta \mathrm{K}$ (log probability of data derived delta K) value [33]. Repeated sampling analysis and the genetic structural plot were performed by CLUMPAK [34]. In this approach, each individual was assigned to populations (named RP1-RP(K) and MIX) based on its maximum membership coefficient using a threshold value of 0.65 for the $Q$ statistic according to the optimum $\mathrm{K}$ value. Each individual was assigned to RPs when the maximum membership coefficient of the individual above 0.65 , otherwise, it was classified into MIX [35]. Unweighted neighbor-joining phylogenetic trees and principal coordinate analyses were both performed based on the dissimilarity matrix calculated with Manhattan index, using DARwin software (version 6.0.9) [36,37]. To summarize the patterns of variation in multilocus dataset, and principal coordinate analysis (PCoA) was also performed using GenAlEx version 6.5 software based on the matrix of pairwise Nei's genetic distance [38].

\subsection{Genetic Diversity Statistics}

The frequency of null alleles (FNA) and scoring errors were estimated using the Microchecker software 2.2.3 [39]. Genetic diversity parameters such as allele frequency, observed number of alleles $(\mathrm{Na})$, effective number of alleles $(\mathrm{Ne})$, expected $(\mathrm{He})$ and observed heterozygosities (Ho), Nei's gene diversity (GD), the percentage of polymorphic loci (PPB), gene flow (Nm), the Shannon's information 
index (I), and Wright's fixation index (F) were calculated using POPGENE v1.3.1 software [40]. The polymorphism information content (PIC) was calculated for each locus using the online program PICcalc [41]. F-statistics, including inbreeding coefficient within individuals ( $\left.\mathrm{F}_{\mathrm{IS}}\right)$, genetic differentiation among populations $\left(\mathrm{F}_{\mathrm{ST}}\right)$, and the pairwise Fst, were computed using GenAlEx version 6.5, which was also conducted the hierarchical analyses of molecular variance (AMOVA) [38]. The Ewens-Watterson test for neutrality at each locus was performed using POPGENE v1.3.1 [40].

\subsection{Construction and Evaluation of the Core Collection}

A core collection-a reduced number of samples which represents the greatest diversity of the initial collection-was generated using PowerCore (v. 1.0) with a heuristic search [30]. The representativeness of the core collection was validated according to the following criteria [22,24,42]. (1) Harboring all alleles present in the entire collection; (2) no significant differences in variability parameters (Ho and $\mathrm{He}$ ) between the two collections, all the comparison were carried out with SPSS v.16.0 (SPSS, Chicago, IL, USA) at significance level below $0.05(p<0.05)$; (3) validating the core collection with unweighted neighbor-joining dissimilarity trees using DARwin software (version 6.0.9) [36]; (4) and a matrix of pairwise Nei's unbiased genetic distance [43] was constructed using POPGENE v1.3.1 software [40], based on which, an Unweighted Pair Group Method with Arithmetic Mean (UPGMA) tree was constructed to reveal the relationship among individuals in the collection using NTSYS-pc software (version 2.1) [44].

\section{Results}

\subsection{Polymorphism of 19 Microsatellite Markers}

Nineteen microsatellite markers were used in the present study (Table S2), a total of 77 alleles were detected across the $251 \mathrm{D}$. odorifera individuals, and the number of alleles detected per locus varied from two to seven (Table 1). The polymorphic information contents (PIC) ranged from 0.03, at S26, to 0.66, at S21, with a mean of 0.32. The mean Shannon's Information index (I), observed (Ho), and expected heterozygosity $(\mathrm{He})$ were $0.65,0.33$, and 0.38 , respectively. Furthermore, null alleles were found at loci S03, S04, S09, S22, S23, S24, S26, and S28. All 19 microsatellite loci were selectively neutral according to the Ewens-Watterson test for neutrality (Table S3).

Table 1. Diversity statistics of the 19 microsatellite markers across Dalbergia odorifera germplasm.

\begin{tabular}{cccccccccccc}
\hline Locus & Size & Na & Ne & Ho & He & GD & PIC & I & Nm & F & FNA \\
\hline S01 & 251 & 4 & 2 & 0.46 & 0.50 & 0.5 & 0.43 & 0.87 & 1.93 & 0.08 & $0.03^{\text {no }}$ \\
S02 & 251 & 3 & 1.06 & 0.05 & 0.06 & 0.06 & 0.06 & 0.15 & 5.63 & 0.11 & $0.0^{\text {no }}$ \\
S03 & 249 & 3 & 1.98 & 0.38 & 0.50 & 0.49 & 0.37 & 0.70 & 2.53 & 0.23 & $0.08^{*}$ \\
S04 & 251 & 4 & 1.64 & 0.26 & 0.39 & 0.39 & 0.36 & 0.72 & 0.44 & 0.35 & $0.1^{\text {* }}$ \\
S07 & 251 & 2 & 1.87 & 0.44 & 0.47 & 0.47 & 0.36 & 0.66 & 3.22 & 0.05 & $0.02^{\text {no }}$ \\
S08 & 251 & 4 & 1.46 & 0.29 & 0.31 & 0.31 & 0.29 & 0.62 & 5.67 & 0.06 & $0.01^{\text {no }}$ \\
S09 & 251 & 4 & 2.39 & 0.45 & 0.58 & 0.58 & 0.53 & 1.08 & 2.60 & 0.23 & $0.08^{*}$ \\
S10 & 250 & 4 & 1.92 & 0.46 & 0.48 & 0.48 & 0.42 & 0.83 & 3.37 & 0.04 & $0.01^{\text {no }}$ \\
S11 & 251 & 2 & 1.62 & 0.36 & 0.38 & 0.38 & 0.31 & 0.57 & 2.49 & 0.05 & $0.01^{\text {no }}$ \\
S12 & 251 & 2 & 1.42 & 0.25 & 0.30 & 0.30 & 0.25 & 0.47 & 2.73 & 0.15 & $0.04^{\text {no }}$ \\
S21 & 250 & 7 & 3.48 & 0.69 & 0.71 & 0.71 & 0.66 & 1.34 & 2.45 & 0.03 & $0.01^{\text {no }}$ \\
S22 & 251 & 6 & 1.28 & 0.19 & 0.22 & 0.22 & 0.21 & 0.53 & 9.45 & 0.13 & $0.02^{*}$ \\
S23 & 251 & 6 & 1.04 & 0.02 & 0.04 & 0.04 & 0.04 & 0.12 & 33.31 & 0.33 & $0.01^{*}$ \\
S24 & 251 & 6 & 2.33 & 0.49 & 0.57 & 0.57 & 0.50 & 0.99 & 1.23 & 0.15 & $0.05^{*}$ \\
S26 & 251 & 3 & 1.03 & 0.02 & 0.03 & 0.03 & 0.03 & 0.08 & 9.53 & 0.28 & $0.01^{*}$ \\
S27 & 251 & 4 & 1.3 & 0.21 & 0.23 & 0.23 & 0.20 & 0.41 & 6.58 & 0.08 & $0.01^{\text {no }}$ \\
S28 & 251 & 2 & 1.7 & 0.29 & 0.41 & 0.41 & 0.33 & 0.6 & 2.18 & 0.29 & $0.08^{*}$ \\
S29 & 251 & 6 & 1.91 & 0.44 & 0.48 & 0.48 & 0.39 & 0.78 & 1.07 & 0.07 & $0.02^{\text {no }}$ \\
S30 & 251 & 5 & 1.92 & 0.43 & 0.48 & 0.48 & 0.40 & 0.79 & 1.68 & 0.11 & $0.04^{\text {no }}$ \\
Mean & 251 & 4.05 & 1.76 & 0.33 & 0.38 & 0.38 & 0.32 & 0.65 & 5.16 & 0.15 & - \\
\hline
\end{tabular}

Size number of individuals with successful amplicons; Na: observed number of alleles; Ne: effective number of alleles; Ho: observed heterozygosity: He: expected heterozygosity; GD: Nei's gene diversity; PIC: polymorphic information content; I: Shannon's Information index; Nm: gene flow, estimated from Fst, Nm = [(1/Fst) - 1$] / 4$; F: fixation index; FNA: frequency of null alleles: ${ }^{\text {no }}$ contained no null allele, ${ }^{*}$ likely contained null alleles $(p<0.05)$. 


\subsection{Population Structure of D. odorifera Germplasm}

An admixture model-based approach was implemented to investigate the population structure of 251 D. odorifera individuals. The optimum cluster was four, which was generated from the STRUCTURE HARVESTER website with the largest $\ln P(\mathrm{D})$-derived $\Delta \mathrm{K}$ (log probability of data derived delta $\mathrm{K}$ ) value (Figure $1 \mathrm{a}-\mathrm{c}$ ). Subsequently, the 251 individuals were classified into five populations based on their maximum membership coefficients, which were designated as RP1 to RP4, and a mixed population MIX (Figure 2). Among these populations (Table 2), MIX contained the largest amount of member 86 including 13 wild ones, followed by RP2 (51, three wild), RP3 (41, 15 wild), RP4 (41, six wild), and RP1 (33, five wild). The information on the geographic origins, types and inferred reconstructed populations is available in Table S1.
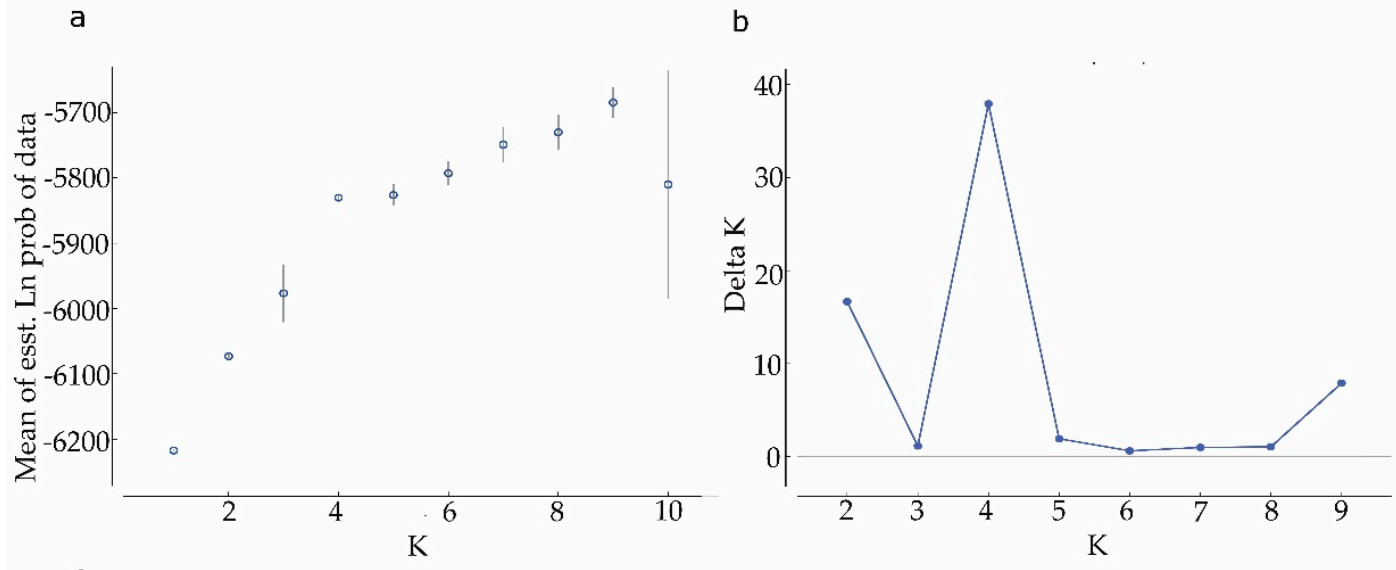

C

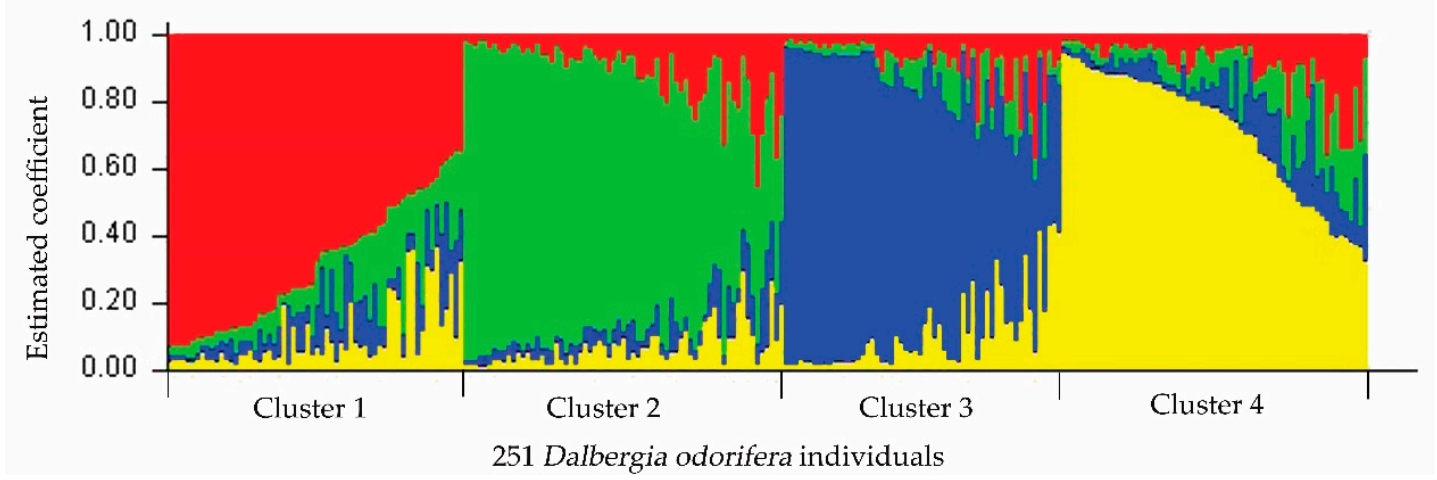

Figure 1. Results of STRUCTURE analysis for 251 Dalbergia odorifera individuals based on microsatellite data. (a) Estimation of population using mean of estimated $\ln \mathrm{P}(\mathrm{D})(\log$ probability of data) with cluster number $(\mathrm{K})$ ranged from one to ten. (b) Estimation of population using $\ln \mathrm{P}(\mathrm{D})$-derived delta $\mathrm{K}$ with cluster number $(\mathrm{K})$ ranged from one to ten. (c) Four estimated clusters of the 251 D. odorifera individuals presented with different colors inferred by STRUCTURE analysis. Clusters 1-4 were presented by red, green, blue, and yellow, respectively. Each bar represented an individual, in which, different color represents the estimated membership coefficients using the $\mathrm{Q}$ statistic. 

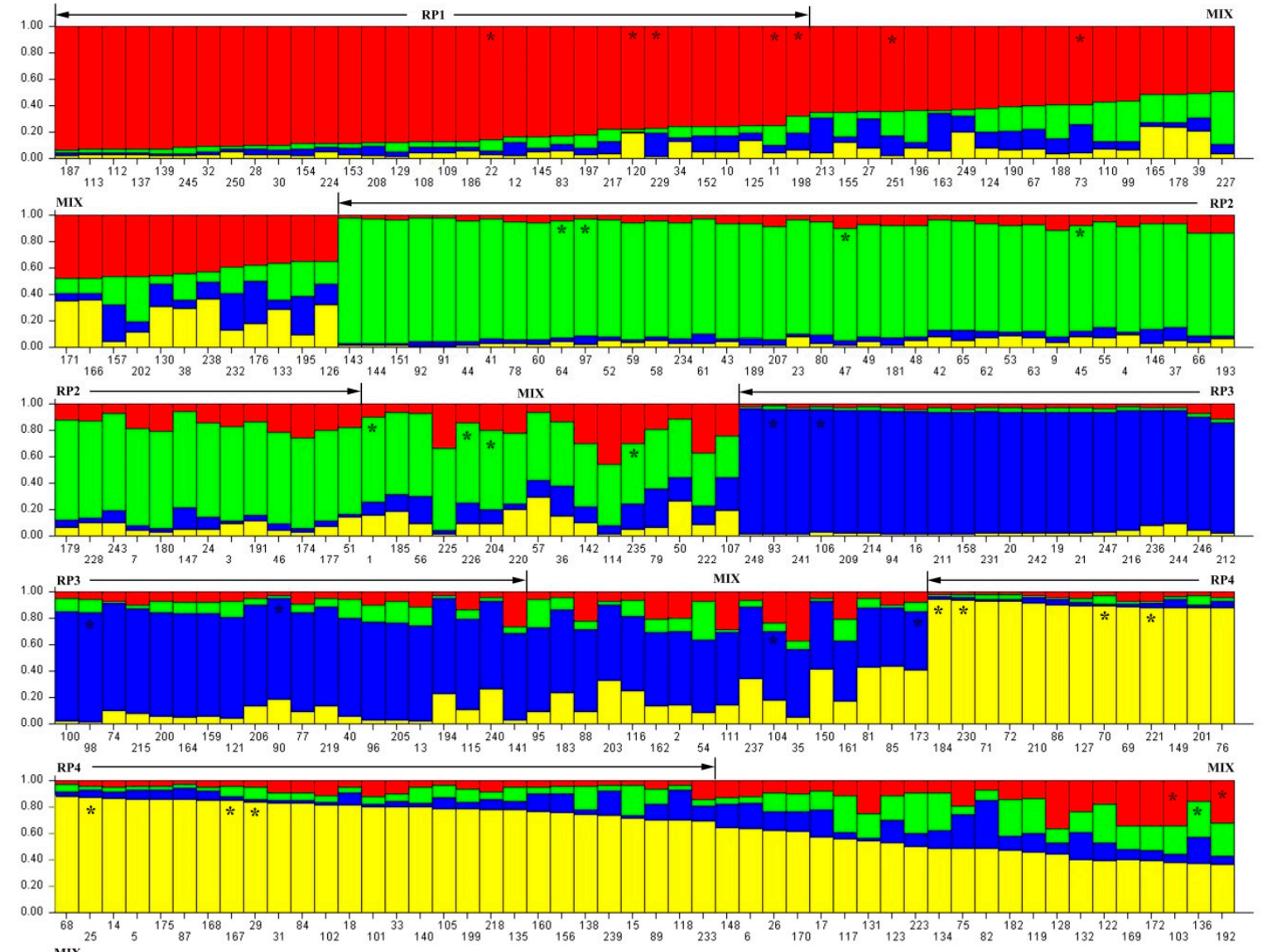

$$
\text { (1.00 }
$$

Figure 2. Details of hierarchal structure analysis on 251 Dalbergia odorifera individuals. RP1, RP2, RP3, RP4, and MIX: population code; *: core collection member; Arabic numerals: ID for each individual, see Table S1.

Table 2. Summary on genetic diversity statistics of Dalbergia odorifera populations.

\begin{tabular}{ccccccccccc}
\hline Population & Size & Wild & Alleles & Na & Ne & Np & Ho & He & GD & PPB \% \\
\hline MIX & 86 & 13 & 66 & 3.47 & 1.74 & 9 & 0.34 & 0.36 & 0.36 & 100.00 \\
RP1 & 32 & 5 & 48 & 2.53 & 1.63 & 2 & 0.34 & 0.33 & 0.32 & 100.00 \\
RP2 & 51 & 3 & 48 & 2.53 & 1.62 & 1 & 0.33 & 0.32 & 0.32 & 94.74 \\
RP3 & 41 & 15 & 53 & 2.79 & 1.55 & 2 & 0.27 & 0.30 & 0.30 & 89.47 \\
RP4 & 41 & 6 & 51 & 2.68 & 1.84 & 3 & 0.33 & 0.38 & 0.37 & 94.74 \\
Mean & 50.2 & 8.4 & 53.2 & 2.80 & 1.68 & - & 0.32 & 0.34 & 0.33 & 95.79 \\
Total $^{\text {a }}$ & 251 & 42 & 77 & 4.05 & 1.76 & - & 0.33 & 0.38 & 0.38 & 100 \\
\hline
\end{tabular}

Population see Figure 2 and Table S1; Size: number of individuals; Wild: number of wild individuals; Alleles: total number of detected alleles; Na: observed mean number of alleles; Ne: mean effective number of alleles; Np: number of private alleles; Ho: observed heterozygosity; He: expected heterozygosity; GD: Nei's gene diversity; PPB \%: the percentage of polymorphic loci; a diversity indices averaged over the 19 loci across all D. odorifera individuals, $\mathrm{b}$ total number of individuals.

Neighbor-joining (NJ) phylogenetic analysis and principal component analysis (PCA) were used to detect the genetic relationship across the 251 individuals based on the dissimilarity matrix calculated with Manhattan index. Four clusters were clearly distinguished by both the NJ dendrogram tree and the PCA plot (Figure 3). Moreover, the $x$ - and y-axis in the PCA plot explained $9.67 \%$ and $8.20 \%$ of variance within the molecular data, respectively. 
a

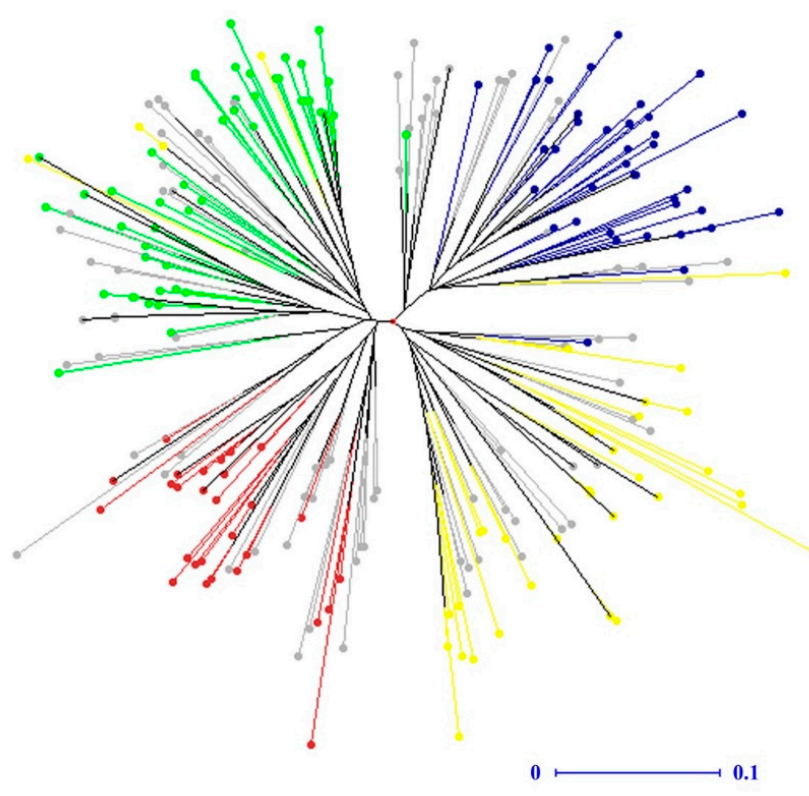

b

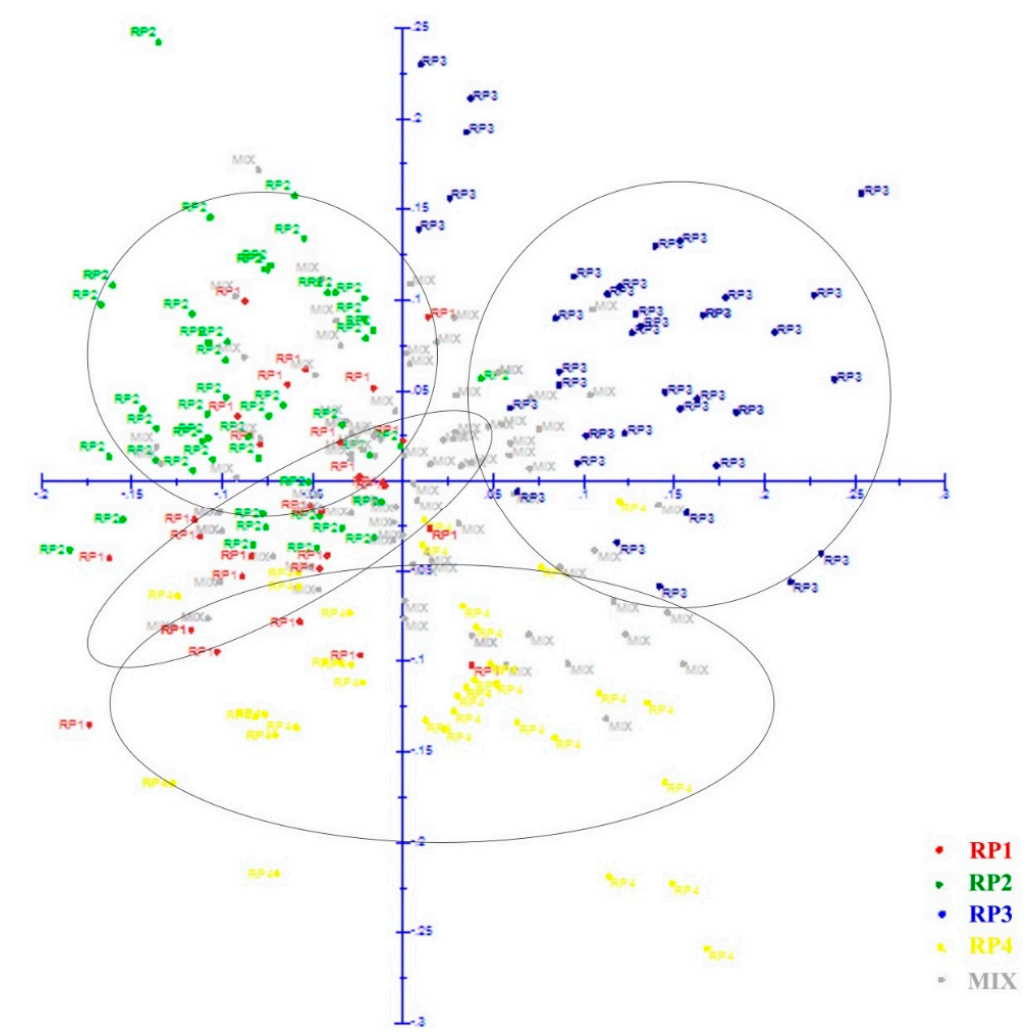

Figure 3. Relationships of the 251 Dalbergia odorifera individuals. (a) An unweighted NJ (neighbor-joining) tree based on the dissimilarity matrix calculated with Manhattan index among 251 D. odorifera individuals. (b) Principal coordinate analysis (PCA) based on the dissimilarity matrix, the $x$-axis, the first principal coordinate explained $9.67 \%$ of variation; the $y$-axis, the second principal coordinate explained $8.20 \%$ of variation. 


\subsection{Genetic Diversity and Variation of D. odorifera Germplasm}

Among the five populations (Figure 2, Table S1), the number of polymorphic loci varied from 17 (RP3) to 19 (MIX and RP1), along with the percentage of polymorphic loci (PPB) from 89.47\% to $100.00 \%$ (Table 2). The largest values of allele (total number of detected alleles), Na (observed mean number of alleles), and Np (number of private alleles) were all detected in MIX, which were 66, 1.74, and 9, respectively. Moreover, RP4 presented the highest genetic diversity among these populations, showing the largest value of expected heterozygosity (He) 0.38 and Nei's gene diversity (GD) 0.37. Additionally, both the expected heterozygosity and Nei's gene diversity was 0.38 within the 251 D. odorifera individuals.

Both analysis of molecular variance (AMOVA) and pairwise Fst analysis were performed to investigate the genetic variations among these populations. The results showed that $11 \%$ of the total genetic variation occurred among populations (Table 3). Moderate genetic differentiation was indicated by pairwise Fst ranging from 0.031 to 0.095 (Table 4). The highest level appeared between RP2 and RP3, whereas the lowest appeared between RP2 and MIX. Furthermore, the principal coordinate analysis (PCoA) was carried out using the GenAlEx version 6.5 based on the matrix of pairwise Nei's unbiased genetic distance. The results showed that $40.11 \%$ of the variance within the molecular data was illustrated by the first axis, and $32.14 \%$ explained by the second axis (Figure S1). Additionally, the five populations could clearly group into three clusters: MIX, RP2, and RP1 represented one cluster, while RP3 and RP4 each represented a cluster.

Table 3. Analysis of molecular variance (AMOVA) for Dalbergia odorifera populations.

\begin{tabular}{cccccc}
\hline Source & d.f. & Sum of Square & Mean of Square & Variance Components & Percentage of Variation \\
\hline Among populations & 4 & 171.788 & 42.947 & 0.408 & $11 \%$ \\
Within populations & 497 & 1621.023 & 6.527 & 3.264 & $89 \%$ \\
Among Individuals & 246 & 844.523 & 3.433 & 0.170 & $5 \%{ }^{* * *}$ \\
Within Individuals & 251 & 776.500 & 3.094 & 3.094 & $84 \%$ \\
Total & 501 & 1792.811 & & 3.671 & $100 \%$ \\
\hline
\end{tabular}

d.f. degrees of freedom, ${ }^{* * *}$ significant of data rand probability, $p<0.001$.

Table 4. The matrix of pairwise genetic differentiation among populations (Fst).

\begin{tabular}{cccccc}
\hline Population & MIX & RP1 & RP2 & RP3 & RP4 \\
\hline MIX & & & & & \\
RP1 & 0.032 & & & & \\
RP2 & 0.031 & 0.065 & & & \\
RP3 & 0.041 & 0.091 & 0.095 & & \\
RP4 & 0.032 & 0.071 & 0.064 & 0.087 & \\
\hline
\end{tabular}

\subsection{Core Collection Development of Dalbergia odorifera}

To conserve an overview for the whole genetic diversity of the germplasm, a core collection that contained $12.4 \%$ of the $251 \mathrm{D}$. odorifera individuals was constructed using the PowerCore software with the advanced M-strategy (Table S1). The core collection included 24 cultivated trees and seven wild trees, and harbored a total of 77 alleles with the observed allele number varying from two to seven per locus (Table S4). The observed number of alleles was 4.05, which was exactly the same as the whole database. Moreover, there was no significant difference on genetic diversity indices between the core collection and the whole database, and the observed and expected heterozygosity was 0.33 and 0.44 , respectively (Table 5). Details on genetic diversity and variations statistics of the core collection were available in Table S5 and Table S6. Moreover, the NJ dendrogram tree showed that the core collection was uniformly distributed in the entire D. odorifera germplasm based on genetic dissimilarity (Figure 4), and based on the matrix of unbiased Nei's genetic distance, the phylogenetic relationships among the 31 individuals was exhibited in the UPGMA tree (Figure 5). 
Table 5. Comparisons on genetic diversity indices between the core collection and the whole Dalbergia odorifera database.

\begin{tabular}{ccccccc}
\hline Population & Size & $\mathbf{N a}^{\mathbf{a}}$ & $\mathbf{N e}^{\mathbf{a}}$ & $\mathbf{H o}^{\mathbf{a}}$ & $\mathbf{H e}^{\mathbf{a}}$ & $\mathbf{G D}^{\mathbf{a}}$ \\
\hline Core collection & 31 & 4.05 & 1.96 & 0.34 & 0.44 & 0.44 \\
Whole database & 251 & 4.05 & 1.76 & 0.33 & 0.38 & 0.38 \\
\hline
\end{tabular}

Size: number of individuals; Na: observed number of alleles; Ne: effective number of alleles; Ho: observed heterozygosity; He: expected heterozygosity; GD: Nei's gene diversity, ${ }^{a}$ no significant difference between the core collection and the whole database, the paired samples $t$-test: $\mathrm{t} 1.848$, Sig. (2-tailed) $0.138, d f: 4$.

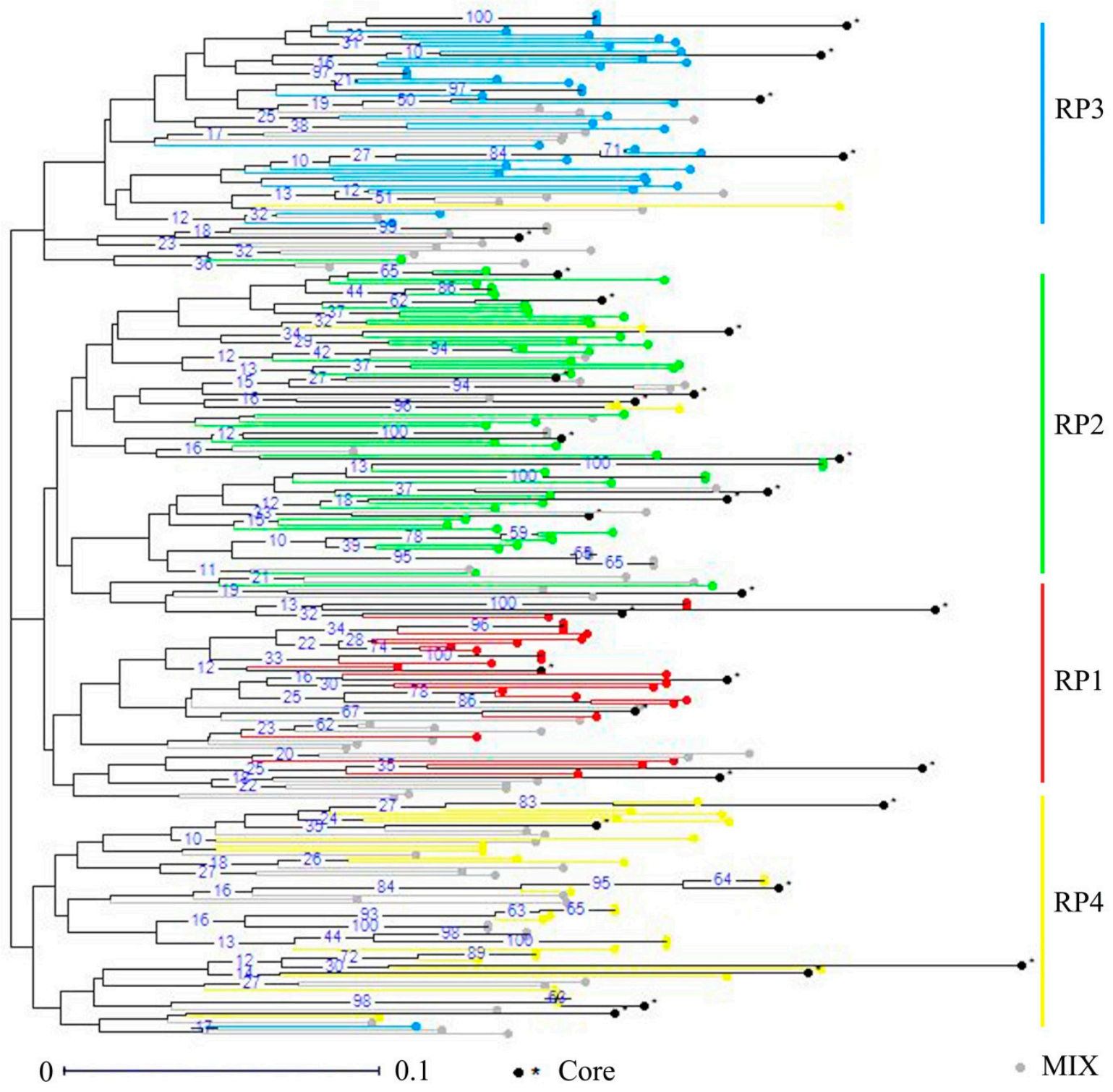

Figure 4. Distribution of the core collection in the NJ tree of the whole database based on genetic dissimilarity. 


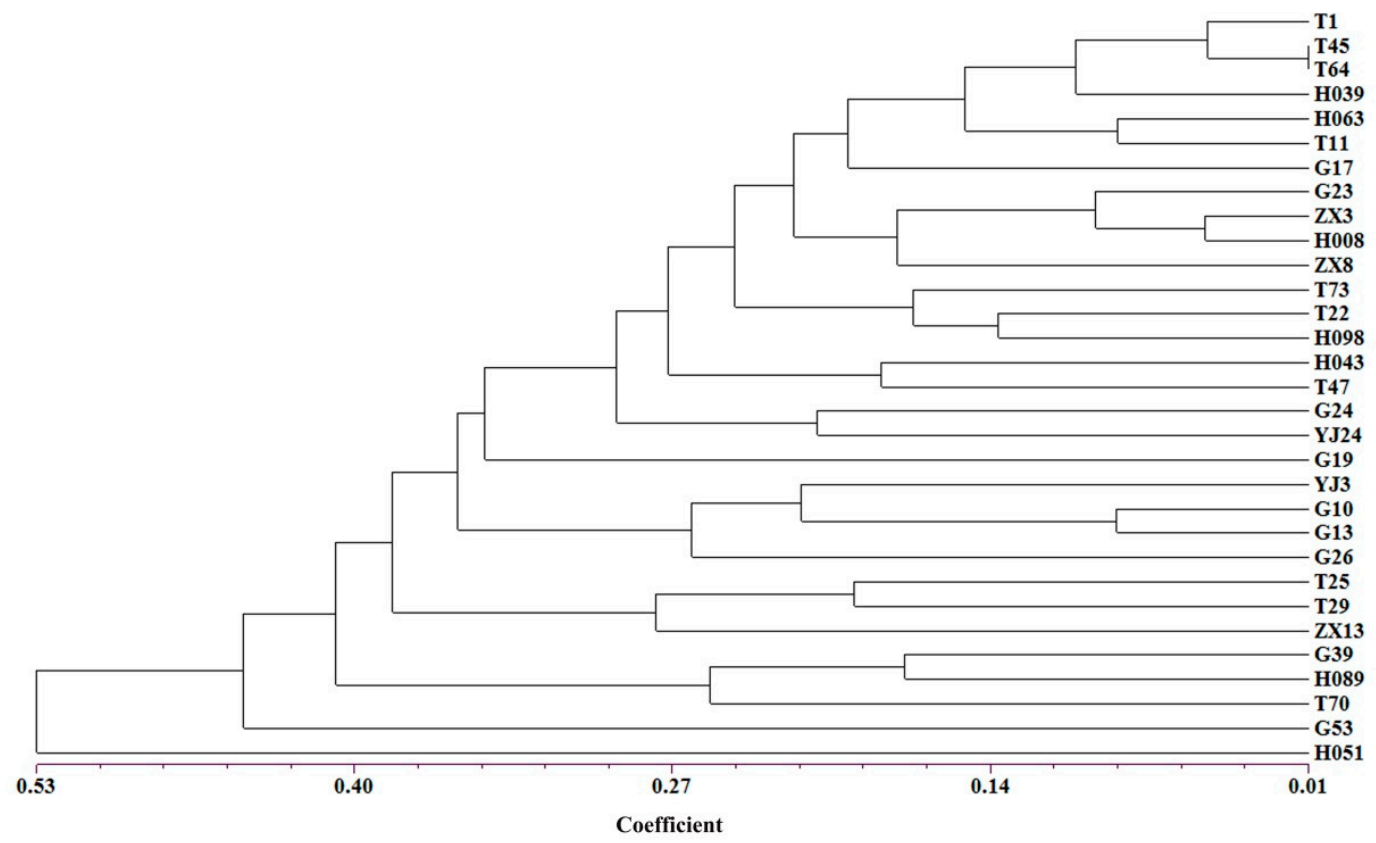

Figure 5. The Unweighted Pair Group Method with Arithmetic Mean (UPGMA) tree of the core collection based on Nei's unbiased genetic distance.

\section{Discussion}

\subsection{Genetic Diversity and Population Structure of D. odorifera Germplasm}

Genetic diversity plays an important role in genetic improvement through breeding programs [45]. However, information on $D$. odorifera genetic diversity is limited. Prior to the present study, only two reports have been available: Yang et al. [11] evaluated genetic diversity of 77 wild D. odorifera trees using six RAPD (random amplified polymorphic DNA) markers and Liu et al. [45] assessed 42 wild trees using 19 SSR (simple sequence repeat) markers. Both reports indicated medium genetic diversity level, which was inferred by Nei's gene diversity value of 0.21 (RAPD) and 0.36 (SSR). Compared to the two studies, a relatively higher genetic diversity level with a higher Nei's gene diversity value 0.38 (Table 1 ) was exhibited in our result on assessing a dataset of $251 \mathrm{D}$. odorifera individuals using 19 microsatellite markers. The difference in genetic diversity may be mainly resulted from the larger population size investigated in the present study [20,46], or alternatively, from the different numbers [47] or types of molecular markers [48] used in these studies.

Assessment on the genetic diversity and population structure of a species is essential to evaluate the applicable potential of a new germplasm resource, and the prior knowledge of genetic diversity and pairwise relatedness can provide beneficial clues for efficient utilization in large collections of genetic resources $[35,49,50]$. Therefore in this study, we assessed the genetic diversity of $251 \mathrm{D}$. odorifera individuals collected from its whole native habitat and introduced sites covering five provinces in southern China. In total, 19 microsatellite markers harbored 77 alleles across the whole database with the mean polymorphic information content (PIC) of 0.32 (Table 1). Medium genetic diversity level of D. odorifera was inferred by Shannon's information index and observed and expected heterozygosity of $0.65,0.33$, and 0.38 , respectively (Table 1 ). The results of genetic diversity indices were comparable to Dalbergia sissoo Roxb. (PIC 0.30) [51], but much lower than these reported for other Dalbergia species, such as Dalbergia monticola Bosser \& R. Rabev. (He 0.83) [52], Dalbergia nigra (Vell.) Benth. (Ho 0.69, He 0.75) [53], Dalbergia cochinchinensis Pierre ex Laness (Ho 0.56, He 0.55), and Dalbergia oliveri Prain (Ho 0.76, He 0.73) [54]. This difference may be attributed to that $D$. odorifera is an endemic tree species with original distribution restricted to the small regions of Hainan Island, which is concordant with the general trend that distribution-restricted plant species are associated with relatively low genetic diversity [55-58]. 
The Bayesian model-based structure analysis is widely used for the inference of hidden population structure in plant species [32]. In this study, structure analysis showed that four was the optimum cluster for the 251 D. odorifera individuals (Figure 1). Both neighbor-joining (NJ) phylogenetic analysis and principal component analysis (PCA) verified the structural pattern as distinctively showing four main clusters (Figure 3). The results of the AMOVA analysis showed that most of the genetic variation was within the populations, while $11 \%$ genetic variation components existed among populations. Similar observations have reported for D. oliveri (12.6\%) [54] and D. sissoo (14.6\%) [59], which may be due to the fact that woody species with a predominately outcrossing tend to have less differentiation among populations and high variation within populations [60].

\subsection{The Core Collection of D. odorifera Germplasm}

Core collections are subsamples of large germplasm collections that include the highest genetic diversity with the minimum number of representative accessions [19]; the development of a core collection in a manageable sized subset will largely reduce redundant labors in the limited breeding cycles and making significant advances in genetic improvement. Therefore, based on 19 neutral selectively microsatellite markers (Table S3), we developed an efficient core collection using the PowerCore software with the advanced M-strategy, associating with the neighbor joining (NJ) analysis and Unweighted Pair Group Method with Arithmetic Mean (UPGMA) analysis based on the matrixes of genetic dissimilarity and Nei's unbiased genetic distance, respectively $[15,20,24,35,61,62]$. The core collection comprised 31 individuals sufficient to retain all the alleles identified from the D. odorifera database (Figure 4; Table S1), of which, the total alleles and observed number of alleles was 77 and 4.05 , respectively, exactly the same as the whole database. Thus, it guaranteed the preservation of alleles, which is essential for maintaining the genetic diversity of a population [63]. Moreover, the observed and expected heterozygosity ( $\mathrm{Ho}$ and $\mathrm{He}$ ) values calculated on the core collection were 0.33 and 0.44 , respectively, showing no significant difference to the whole database, neither did the other genetic diversity indices (Table 5). In the core collection, the observed heterozygosity of the five populations varied from 0.25 (RP3) to 0.40 (RP1), the expected heterozygosity varied from 0.36 (RP2) to 0.44 (RP4) (Table S5), and the values of pairwise Fst (genetic differentiation among populations) from 0.052, between MIX and RP2, to 0.170, between RP3 and RP2 (Table S6). These findings demonstrated that the core collection represented sufficient genetic variation of the whole database. Additionally, the members of the core collection distributed evenly among the $251 \mathrm{D}$. odorifera individuals were validated by the results of the NJ and UPGMA analysis (Figures 4 and 5).

As a whole, the 31 genotypes selected for the core collection are representative samples of the diversity retained from the whole southern China that covering its native habitat and the whole first introduced sites. Notably, the wild resource of $D$. odorifera is highly endangered and rare due to the overexploitation, and their distributions in their original habitat (Hainan Island) are severely fragmented but relationships among individuals are badly influenced by human activities. It is no wonder then that the core collection comprised only six wild individuals compared to 25 cultivated ones.

Prior to the present study, no information has been reported on core collection of $D$. odorifera. Therefore, this core collection can serve a basic reference on similar research for D. odorifera and other Dalbergia species. Furthermore, it can be considered as a powerful tool for exploring the genetic diversity, as well as a new source for efficient conservation and breeding programs of $D$. odorifera in the future.

\section{Conclusions}

The present study provides an overall assessment on genetic diversity and structure of 251 D. odorifera germplasm. A medium level of genetic diversity and genetic variation was presented within the species. Based on the assessment, a core collection was first established using the PowerCore software with the M-strategy. The core collection contained $12.4 \%$ (31) out of the 251 germplasm, possessing the intact genetic diversity of the whole germplasm collection. This core collection will 
serve as a primary source for further genetic association, functional analyses, and function to improve breeding programs in future studies.

Supplementary Materials: The following are available online at http://www.mdpi.com/2073-4425/10/4/281/s1, Figure S1: Principal coordinate analysis (PCoA) based on pairwise Nei's unbiased genetic distance. Table S1: Information of 251 Dalbergia odorifera germplasm, Table S2: Details of 19 microsatellite markers, Table S3: The overall Ewens-Watterson test for neutrality across 19 microsatellite loci in Dalbergia odorifera germplasm, Table S4: Diversity statistics of the 19 microsatellite markers across the core collection of 30 Dalbergia odorifera individuals, Table S5: Summary on genetic diversity statistics of Dalbergia odorifera core collection, Table S6: Pairwise genetic differentiation index (Fst) values in core collection.

Author Contributions: Data Curation, N.-N.Z. and Z.-J.Y.; Formal Analysis, X.-J.L. and H.-Y.J.; Funding Acquisition, N.-N.Z. and D.-P.X.; Investigation, X.-J.L. and Z.-J.Y.; Project Administration, X.-J.L. and D.-P.X.; Resources, N.-N.Z., Z.-J.Y., and H.-Y.J.; Supervision, D.-P.X.; Writing-Original Draft, F.L.; Writing一Review \& Editing, F.-M.L.

Funding: This work was funded by grants from the Research Funds for the Central Non-profit Research Institution of Chinese Academy of Forestry (CAFYBB2017ZX001-4), the Science Innovation Projects of Guangdong Province (2016KJCX009), and the National Natural Science Foundation of China (31500537). The APC was funded by CAFYBB2017ZX001-4.

Acknowledgments: The authors are very grateful to Chun-Jie Fang and Zi-Yi Cui (Research Institute of Tropical Forestry, Chinese Academy of Forestry) for their helpful comments and support on this manuscript.

Conflicts of Interest: The authors declare no conflict of interests.

\section{References}

1. Fan, Z.M.; Wang, D.Y.; Yang, J.M.; Lin, Z.X.; Lin, Y.X.; Yang, A.L.; Fan, H.; Cao, M.; Yuan, S.Y.; Liu, Z.J.; et al. Dalbergia odorifera extract promotes angiogenesis through upregulation of VEGFRs and PI3K/MAPK signaling pathways. J. Ethnopharmacol. 2017, 204, 132-141. [CrossRef] [PubMed]

2. Lee, D.S.; Li, B.; Keo, S.; Kim, K.S.; Jeong, G.S.; Oh, H.; Kim, Y.C. Inhibitory effect of 9-hydroxy-6,7dimethoxydalbergiquinol from Dalbergia odorifera on the NF-kappaB-related neuroinflammatory response in lipopolysaccharide-stimulated mouse BV2 microglial cells is mediated by heme oxygenase-1. Int. Immunopharmacol. 2013, 17, 828-835. [CrossRef] [PubMed]

3. Choi, H.S.; Park, J.A.; Hwang, J.S.; Ham, S.A.; Yoo, T.; Lee, W.J.; Paek, K.S.; Shin, H.C.; Lee, C.H.; Seo, H.G. A Dalbergia odorifera extract improves the survival of endotoxemia model mice by inhibiting HMGB1 release. BMC Complement. Altern. Med. 2017, 17, 212. [CrossRef] [PubMed]

4. The, S.N. A review on the medicinal plant Dalbergia odorifera species: Phytochemistry and biological activity. Evid.-Based Complement. Altern. Med. 2017, 2017,1-27. [CrossRef]

5. Lee, D.S.; Kim, K.S.; Ko, W.; Li, B.; Keo, S.; Jeong, G.S.; Oh, H.; Kim, Y.C. The neoflavonoid latifolin isolated from $\mathrm{MeOH}$ extract of Dalbergia odorifera attenuates inflammatory responses by inhibiting NF-kappaB activation via Nrf2-mediated heme oxygenase-1 expression. Phytother. Res. 2014, 28, 1216-1223. [CrossRef] [PubMed]

6. Wang, H.; Dong, W.H.; Zuo, W.J.; Wang, H.; Zhong, H.M.; Mei, W.L.; Dai, H.F. Three new phenolic compounds from Dalbergia odorifera. J. Asian Nat. Prod. Res. 2014, 16, 1109-1118. [CrossRef]

7. Meng, H.; Chen, D.L.; Yang, Y.; Liu, Y.Y.; Wei, J.H. Sesquiterpenoids with cytotoxicity from heartwood of Dalbergia odorifera. J. Asian Nat. Prod. Res. 2018, 1-7. [CrossRef]

8. Tao, Y.; Wang, Y. Bioactive sesquiterpenes isolated from the essential oil of Dalbergia odorifera T. Chen. Fitoterapia 2010, 81, 393-396. [CrossRef]

9. Wang, H.; Dong, W.H.; Zuo, W.J.; Liu, S.; Zhong, H.M.; Mei, W.L.; Dai, H.F. Five new sesquiterpenoids from Dalbergia odorifera. Fitoterapia 2014, 95, 16-21. [CrossRef] [PubMed]

10. So, T.; Dell, B. Conservation and utilization of threatened hardwood species through reforestation-An example of Afzelia xylocarpa (Kruz.) craib and Dalbergia cochinchinensis Pierre in Cambodia. Pac. Conserv. Biol. 2010, 16, 101-116. [CrossRef]

11. Yang, Q.X.; Feng, J.D.; Wei, J.H.; Li, R.T.; He, M.J. Genetic diversity of China' s endangered medicinal plant Dalbergia odorifera. World Sci. Technol. Mod. Tradit. Chin. Med. Mater. Medica 2007, 9, 73-79.

12. World Conservation Monitoring Centre. Dalbergia odorifera. The IUCN Red List of Threatened Species. 1998: e.T32398A9698077. Available online: http://www.iucnredlist.org/details/32398/0 (accessed on 18 July 2018). 
13. Liu, F.-M.; Hong, Z.; Yang, Z.-J.; Zhang, N.-N.; Liu, X.-J.; Xu, D.-P. De novo transcriptome analysis of Dalbergia odorifera and transferability of SSR markers developed from the transcriptome. Forests 2019, 10, 98. [CrossRef]

14. Liu, X.; Xu, D.; Yang, Z.; Zhang, N. Geographic variations in seed germination of Dalbergia odorifera T. Chen in response to temperature. Ind. Crop Prod. 2017, 102, 45-50. [CrossRef]

15. You, F.M.; Jia, G.; Xiao, J.; Duguid, S.D.; Rashid, K.Y.; Booker, H.M.; Cloutier, S. Genetic Variability of 27 Traits in a Core Collection of Flax (Linum usitatissimum L.). Front. Plant Sci. 2017, 8, 1636. [CrossRef]

16. Xu, D.P.; Yang, Z.J.; Liang, K.N.; Zhang, N.N.; Zeng, J. Investigation on cold injuries of five valuable tree species in southern China. Sci. Silvae Sin. 2008, 44, 1-2.

17. Yang, Z.J.; Xu, D.P.; Zeng, J.; Guo, J.Y.; Lin, Q.J.; Yao, Q.D. Investigation on cold injuries for Pterocarpus macrocarpus Kurz in southern China. Sci. Silvae Sin. 2008, 44, 123-127.

18. Ma, Y.H.; Jia, R.F.; Zeng, J.; Xu, D.P.; Zhang, N.N.; Liu, X.J. Early growth evaluation on six-year-old Dalbergia odorifera T. Chen families. J. Cent. South Univ. For. Technol. 2017, 37, 42-47.

19. Frankel, O.H. Genetic Perspectives of Germplasm Conservation. Available online: http://books.google.fr/books? $\mathrm{hl}=$ en\&lr=\&id=0yRUkeJFwAMC\&oi=fnd\&pg=PA161\&dq=genetic+erosion+frankel\&ots=XXbSegnxMS\&sig= MbO4m1MOa5npb7zEbebw1XuGiRY) (accessed on 30 January 2019).

20. Lee, H.-Y.; Ro, N.-Y.; Jeong, H.-J.; Kwon, J.-K.; Jo, J.; Ha, Y.; Jung, A.; Han, J.-W.; Venkatesh, J.; Kang, B.-C. Genetic diversity and population structure analysis to construct a core collection from a large Capsicum germplasm. BMC Genet. 2016, 17. [CrossRef]

21. Mariano, L.C.; Zchonski, F.L.; da Silva, C.M.; Da-Silva, P.R. Genetic variability in a Brazilian apple germplasm collection with low chilling requirements. Peer J. 2019, 6, e6265. [CrossRef]

22. Liang, W.; Dondini, L.; De Franceschi, P.; Paris, R.; Sansavini, S.; Tartarini, S. Genetic diversity, population structure and construction of a core collection of apple cultivars from Italian germplasm. Plant Mol. Biol. Rep. 2015, 33, 458-473. [CrossRef]

23. Guzman, L.F.; Machida-Hirano, R.; Borrayo, E.; Cortes-Cruz, M.; Espindola-Barquera, M.D.; Heredia Garcia, E. Genetic structure and selection of a core collection for long term conservation of avocado in Mexico. Front. Plant Sci. 2017, 8, 243. [CrossRef] [PubMed]

24. Pereira-Lorenzo, S.; Ramos-Cabrer, A.M.; Barreneche, T.; Mattioni, C.; Villani, F.; Díaz-Hernández, M.B.; Martín, L.M.; Martín, Á. Database of European chestnut cultivars and definition of a core collection using simple sequence repeats. Tree Genet. Genomes 2017, 13, 114. [CrossRef]

25. Ditta, A.; Zhou, Z.; Cai, X.; Wang, X.; Okubazghi, K.W.; Shehzad, M.; Xu, Y.; Hou, Y.; Sajid Iqbal, M.; Khan, M.K.R.; et al. Assessment of genetic diversity, population structure, and evolutionary relationship of uncharacterized genes in a novel germplasm collection of diploid and allotetraploid Gossypium accessions using EST and genomic SSR markers. Int. J. Mol. Sci. 2018, 19, 2401. [CrossRef]

26. Gomes, S.; Martins-Lopes, P.; Lopes, J.; Guedes-Pinto, H. Assessing genetic diversity in Olea europaea L. using ISSR and SSR markers. Plant Mol. Biol. Rep. 2009, 27, 365-373. [CrossRef]

27. Boraks, A.; Broders, K.D. Population genetic diversity of the rare hardwood butternut (Juglans cinerea) in the northeastern USA. Tree. Genet. Genomes 2016, 12. [CrossRef]

28. Nybom, H. Comparison of different nuclear DNA markers for estimating intraspecific genetic diversity in plants. Mol. Ecol. 2004, 13, 1143-1155. [CrossRef]

29. Kong, Q.; Chen, J.; Yong, L.; Ma, Y.; Peng, L.; Wu, S.; Yuan, H.; Bie, Z. Genetic diversity of Cucurbita rootstock germplasm as assessed using simple sequence repeat markers. Sci. Hortic.-Amsterdam 2014, 175, 150-155. [CrossRef]

30. Kim, K.W.; Chung, H.K.; Cho, G.T.; Ma, K.H.; Chandrabalan, D.; Gwag, J.G.; Kim, T.S.; Cho, E.G.; Park, Y.J. PowerCore: A program applying the advanced $\mathrm{M}$ strategy with a heuristic search for establishing core sets. Bioinformatics 2007, 23, 2155-2162. [CrossRef]

31. Evanno, G.; Regnaut, S.; Goudet, J. Detecting the number of clusters of individuals using the software structure: A simulation study. Mol. Ecol. 2010, 14, 2611-2620. [CrossRef]

32. Porras-Hurtado, L.; Ruiz, Y.; Santos, C.; Phillips, C.; Carracedo, A.; Lareu, M.V. An overview of STRUCTURE: Applications, parameter settings, and supporting software. Front. Genet. 2013, 4, 98. [CrossRef]

33. Earl, D.A.; Vonholdt, B.M. STRUCTURE HARVESTER: A website and program for visualizing STRUCTURE output and implementing the Evanno method. Conserv. Genet. Resour. 2012, 4, 359-361. [CrossRef] 
34. Kopelman, N.M.; Jonathan, M.; Mattias, J.; Rosenberg, N.A.; Itay, M. Clumpak: A program for identifying clustering modes and packaging population structure inferences across K. Mol. Ecol. Resour. 2015, 15, 1179-1191. [CrossRef]

35. Xu, Q.; Zeng, X.; Lin, B.; Li, Z.; Yuan, H.; Wang, Y.; Tashi, N. A microsatellite diversity analysis and the development of core-set germplasm in a large hulless barley (Hordeum vulgare L.) collection. BMC Genet. 2017, 18, 102. [CrossRef] [PubMed]

36. Perrier, X.; Jacquemoud-Collet, J.P. DARwin Software. 2006. Available online: http://darwin.cirad.fr/darwin (accessed on 30 January 2019).

37. Perrier, X.; Flori, A.; Bonnot, F. Data analysis methods. In Genetic Diversity of Cultivated Tropical Plants; Hamon, P., Seguin, M., Perrier, X., Glaszmann, J.C., Eds.; Enfield, Science Publishers: Montpellier, France, 2003; pp. 43-76.

38. Peakall, R.; Smouse, P.E. GenAlEx 6.5: Genetic analysis in Excel. Population genetic software for teaching and research-An update. Bioinformatics 2012, 28, 2537-2539. [CrossRef]

39. Van Oosterhout, C.; Hutchinson, W.F.; Wills, D.P.M.; Shipley, P. Micro-checker: Software for identifying and correcting genotyping errors in microsatellite data. Mol. Ecol. Notes 2004, 4, 535-538. [CrossRef]

40. Popgene, Version 1.32; the User-Friendly Shareware for Population Genetic Analysismolecular Biology and Biotechnology Center, University of AlbertaEdmonton. Available online: http://www.ualberta.ca/ \{\}fyeh (accessed on 23 November 2017).

41. Nagy, S.; Poczai, P.; Cernák, I.; Gorji, A.M.; Hegedús, G.; Taller, J. PICcalc: An online program to calculate polymorphic information content for molecular genetic studies. Biochem. Genet. 2012, 50, 670-672. [CrossRef]

42. Odong, T.L.; Jansen, J.; Van Eeuwijk, F.A.; Van Hintum, T.J.L. Quality of core collections for effective utilisation of genetic resources review, discussion and interpretation. Theor. Appl. Genet. 2013, 126, 289-305. [CrossRef]

43. Jansen, J.; van Hintum, T. Genetic distance sampling: A novel sampling method for obtaining core collections using genetic distances with an application to cultivated lettuce. Theor. Appl. Genet. 2007, 114, 421-428. [CrossRef]

44. Rohlf, F.J. NTSYS-pc: Microcomputer Programs for Numerical Taxonomy and Multivariate Analysis. Am. Stat. 1987, 41, 330. [CrossRef]

45. Liu, F.-M.; Hong, Z.; Xu, D.-P.; Jia, H.-Y.; Zhang, N.-N.; Liu, X.-J.; Yang, Z.-J.; Lu, M.-Z. Genetic diversity of endangered endemic Dalbergia odorifera revealed by SSR markers. Forests 2019, 10, 225. [CrossRef]

46. White, T.L.; Adams, W.T.; Neale, D.B. Forest Genetics; CABI Publishing: Boston, MA, USA, 2007; pp. $149-186$.

47. Ferrer, M.M.; Eguiarte, L.E.; Montana, C. Genetic structure and outcrossing rates in Flourensia cernua (Asteraceae) growing at different densities in the South-western Chihuahuan Desert. Ann. Bot. 2004, 94, 419-426. [CrossRef]

48. Powell, W.; Morgante, M.; Andre, C.; Hanafey, M.; Vogel, J.; Tingey, S.; Rafalski, A. The comparison of RFLP, RAPD, AFLP and SSR (microsatellite) markers for germplasm analysis. Mol. Breed. 1996, 2, 225-238. [CrossRef]

49. Yang, X.; Song, J.; Todd, J.; Peng, Z.; Paudel, D.; Luo, Z.; Ma, X.; You, Q.; Hanson, E.; Zhao, Z.; et al. Target enrichment sequencing of 307 germplasm accessions identified ancestry of ancient and modern hybrids and signatures of adaptation and selection in sugarcane (Saccharum spp.), a 'sweet' crop with 'bitter' genomes. Plant Biotechnol. J. 2019, 17, 488-498. [CrossRef]

50. Ambreen, H.; Kumar, S.; Kumar, A.; Agarwal, M.; Jagannath, A.; Goel, S. Association mapping for important agronomic traits in Safflower (Carthamus tinctorius L.) core collection using microsatellite markers. Front. Plant Sci. 2018, 9, 402. [CrossRef] [PubMed]

51. Bakshi, M.; Sharma, A. Assessment of genetic diversity in Dalbergia sissoo clones through RAPD profiling. J. For. Res. 2011, 22, 393. [CrossRef]

52. Andrianoelina, O.; Rakotondraoelina, H.; Ramamonjisoa, L.; Maley, J.; Danthu, P.; Bouvet, J.-M. Genetic diversity of Dalbergia monticola (Fabaceae) an endangered tree species in the fragmented oriental forest of Madagascar. Biodivers. Conserv. 2006, 15, 1109-1128. [CrossRef]

53. Buzatti, R.S.D.O.; Ribeiro, R.A.; Filho, J.P.D.L.; Lovato, M.B. Fine-scale spatial genetic structure of Dalbergia nigra (Fabaceae), a threatened and endemic tree of the Brazilian Atlantic forest. Genet. Mol. Biol. 2012, 35, 838-846. [CrossRef] [PubMed] 
54. Hartvig, I.; So, T.; Changtragoon, S.; Tran, H.T.; Bouamanivong, S.; Theilade, I.; Kjær, E.D.; Nielsen, L.R. Population genetic structure of the endemic rosewoods Dalbergia cochinchinensis and D. oliveri at a regional scale reflects the Indochinese landscape and life-history traits. Ecol. Evol. 2018, 8, 530-545. [CrossRef] [PubMed]

55. Kang, S.S.; Chung, M.G. Genetic variation and population structure in Korean endemic species: IV. Hemerocallis hakuunensis (Liliaceae). J. Plant Res. 1997, 110, 209-217. [CrossRef]

56. Zhong, T.; Zhao, G.; Lou, Y.; Lin, X.; Guo, X. Genetic diversity analysis of Sinojackia microcarpa, a rare tree species endemic in China, based on simple sequence repeat markers. J. For. Res. 2018. [CrossRef]

57. Gichira, A.W.; Li, Z.-Z.; Saina, J.K.; Hu, G.-W.; Gituru, R.W.; Wang, Q.-F.; Chen, J.-M. Demographic history and population genetic structure of Hagenia abyssinica (Rosaceae), a tropical tree endemic to the Ethiopian highlands and eastern African mountains. Tree Genet. Genomes 2017, 13, 72. [CrossRef]

58. Hamrick, J.L.; Godt, M.J.W.; Sherman-Broyles, S.L. Factors influencing levels of genetic diversity in woody plant species. New For. 1992, 6, 95-124. [CrossRef]

59. Wang, B.Y.; Shi, L.; Ruan, Z.Y.; Deng, J. Genetic diversity and differentiation in Dalbergia sissoo (Fabaceae) as revealed by RAPD. Genet. Mol. Res. 2011, 10, 114. [CrossRef]

60. Li, X.; Li, M.; Hou, L.; Zhang, Z.; Pang, X.; Li, Y. De novo transcriptome assembly and population genetic analyses for an endangered chinese endemic Acer miaotaiense (Aceraceae). Genes 2018, 9, 378. [CrossRef]

61. Li, J.; Liu, X.-B.; Zhao, Z.-W.; Yang, Z.L. Genetic diversity, core collection and breeding history of Pleurotus ostreatus in China. Mycoscience 2019, 60, 14-24. [CrossRef]

62. Yu, J.Z.; Fang, D.D.; Kohel, R.J.; Ulloa, M.; Hinze, L.L.; Percy, R.G.; Zhang, J.; Chee, P.; Scheffler, B.E.; Jones, D.C. Development of a core set of SSR markers for the characterization of Gossypium germplasm. Euphytica 2012, 187, 203-213. [CrossRef]

63. Bengtsson, B.O.; Weibull, P.; Ghatnekar, L. The Loss of Alleles by Sampling: A study of the common outbreeding grass Festuca ovina over three geographic scales. Hereditas 1995, 122, 221-238. [CrossRef]

(C) 2019 by the authors. Licensee MDPI, Basel, Switzerland. This article is an open access article distributed under the terms and conditions of the Creative Commons Attribution (CC BY) license (http://creativecommons.org/licenses/by/4.0/). 